\title{
Estimating forest area at the year 1990 by two-phase sampling on historical remotely sensed imagery in Italy
}

\begin{abstract}
Forest area in the year 1990 is a figure of great interest under the Kyoto Protocol. This note is devoted to a scientific exercise for the probabilistic ex post assessment of such a figure in Italy. Estimation was performed by twophase point sampling, which made use of historical remotely sensed imagery. In the first phase, a sample of 12089 points was selected according to an unaligned systematic sampling and the selected points were classified in land-use categories by Landsat 5 TM imagery. In the second phase, a sample of 3000 points was selected by stratified sampling in which the strata were determined by the satellite classification and the selected points were classified by aerial photos, assumed as ground truth. A two-phase estimate of land-use coverage partitioning the Italian territory was obtained together with a conservative estimate of the sampling variance-covariance. The procedure has proved to be of relatively easy implementation and objective repeatability.
\end{abstract}

Key words Forest area assessment - Point sampling and classification · Two-phase sampling · Unaligned systematic sampling

\section{Introduction}

Forest area in the year 1990 is a figure of great interest in view of reporting requirements of the Kyoto Protocol (under United Nations Framework Convention on Climate Changes, UN-FCCC). The year 1990 is taken as the base year for assessing reductions/emissions of greenhouse gases due to afforestation, reforestation, deforestation, and revegetation during the first commitment period (20082012). Afforestation includes the natural expansion of forests, provided that it can be demonstrated that such an expansion is driven by targeted agricultural/environmental planning measures.

Reliable data about forest area in 1990 are often not available for wide territories (e.g., on a national scale). In such cases, methods for the ex post assessment of forest area may be based on historical remotely sensed imagery.

In this context, the aim of this note is to show and discuss, as an exercise case study, a probabilistic estimation of the 1990 forest area in Italy, on the basis of a two-phase point sampling that makes use of historical satellite and aerial images. As a by-product, the obtained results provide insight into the performance of the statistical procedure for land-use coverage estimation proposed by Fattorini et al. (2004) on a real large-scale situation.

\section{Materials and methods}

Historical remotely sensed images for the ex post assessment of forest area must have an appropriate spatial resolution to grasp the adopted definition of "forest." The Kyoto Protocol definition involves a minimum forest size not larger than 1 ha, and units as small as 1 ha can be identified by sensor systems with a spatial resolution not lower than $30 \mathrm{~m}$ (IPCC 2003). In Italy, minimum forest size, under the nomenclature system officially adopted by the Government for Kyoto Protocol reporting and for the National Forest Inventory, is 0.5 ha (i.e., the same size threshold as defined 